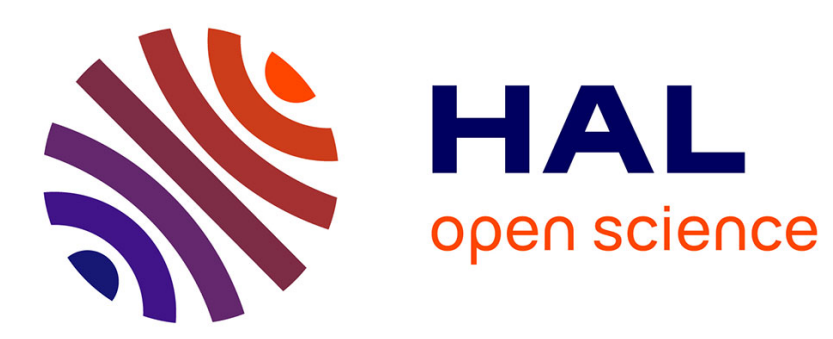

\title{
Subspace Projectors for State-Constrained Multi-Robot Consensus
}

Fabio Morbidi

\section{To cite this version:}

Fabio Morbidi. Subspace Projectors for State-Constrained Multi-Robot Consensus. IEEE International Conference on Robotics and Automation, May 2020, Paris, France. hal-02877294

\section{HAL Id: hal-02877294 \\ https://hal.science/hal-02877294}

Submitted on 22 Jun 2020

HAL is a multi-disciplinary open access archive for the deposit and dissemination of scientific research documents, whether they are published or not. The documents may come from teaching and research institutions in France or abroad, or from public or private research centers.
L'archive ouverte pluridisciplinaire HAL, est destinée au dépôt et à la diffusion de documents scientifiques de niveau recherche, publiés ou non, émanant des établissements d'enseignement et de recherche français ou étrangers, des laboratoires publics ou privés. 


\title{
Subspace Projectors for State-Constrained Multi-Robot Consensus
}

\author{
Fabio Morbidi
}

\begin{abstract}
In this paper, we study the state-constrained consensus problem and introduce a new family of distributed algorithms based on subspace projection methods which are simple to implement and which preserve, under some suitable conditions, the consensus value of the original discrete-time agreement protocol. The proposed theory is supported by extensive numerical experiments for the constrained 2D rendezvous of single-integrator robots.
\end{abstract}

\section{INTRODUCTION}

\section{A. Motivation and related work}

Over the last decade, the consensus protocol [1]-[3] has enjoyed growing success in robotics, and it has been widely employed as a basic building block to design distributed coordination strategies for multi-agent systems. Applications include rendezvous [4], [5], flocking [6]-[8], formation control [9], target encirclement [10], deployment and area monitoring [11], [12], containment and connectivity control [13], [14], and decentralized estimation [15], [16], just to mention the most relevant ones.

However, in many practical situations, cooperative agents are not allowed to freely agree upon a common value, and their state variables are constrained within a given (convex) set. This happens, for example, when multiple mobile robots evolve in confined obstacle-ridden environments, when they are subject to motion constraints (e.g. their velocity is bounded), or they have a limited communication/sensing range. The resulting constrained consensus problem has been investigated from different angles in [17]-[22]. In fact, depending on the nature of constraints (linear vs. nonlinear, equality vs. inequality, including saturations), problems of different complexity arise. A recurring idea in [17] (discrete-time formulation) and in [19], [20] (continuous-time formulation), has been to project the state vector of each agent onto its constraint set. Similar projection techniques have also been used for distributed optimization [21], [23], and more recently, to study the synchronization of a network of Kuramoto oscillators [24].

\section{B. Original contributions, organization and notation}

Inspired by the vast literature on Kalman filtering with state constraints [25], [26], in this paper we show that under suitable conditions, it is possible to constrain the joint state of a multi-agent system within a prescribed linear subspace, without affecting the consensus value of the corresponding unconstrained agreement protocol. In fact, differently from [17], [19], where the local state of each agent is kept inside a closed convex set known only to it, we adopt here an alternative approach based on subspace projectors which presents several attractive features: it is straightforward to

F. Morbidi is with the MIS Laboratory, Université de Picardie Jules Verne, 33 rue Saint-Leu, 80039 Amiens, France. Email: fabio.morbidi@u-picardie.fr implement and it leads to a new class of discrete-time constrained consensus protocols whose group decision properties can be easily characterized thanks to a well-established projection theory [27], [28]. Our theoretical analysis is paired with numerical experiments for the constrained rendezvous problem in the $2 \mathrm{D}$ space.

The rest of this paper is organized as follows. In Sect. II, we briefly review the discrete-time consensus protocol and recall some basic facts about projectors. Sect. III presents the main theoretical results of the paper. The theory is validated with the aid of numerical experiments in Sect. IV. Finally, in Sect. V, we summarize the main contributions of the paper and outline some possible avenues for future research.

Notation: Throughout this paper, $\mathbf{I}_{n}$ denotes the $n \times n$ identity matrix, $\mathbf{0}_{n}$ the $n \times n$ matrix of zeros, $\|\mathbf{x}\|_{2}$ the Euclidean norm of vector $\mathbf{x} \in \mathbb{R}^{n}, \mathbf{x}^{H}$ the conjugate (or Hermitian) transpose of $\mathbf{x}, \otimes$ the Kronecker product, and $\operatorname{Im}(\mathbf{A})$ and $\operatorname{ker}(\mathbf{A})$ the image and null space of matrix $\mathbf{A}$, respectively. $\mathbf{S O}(n) \triangleq\left\{\mathbf{R} \in \mathbb{R}^{n \times n}: \mathbf{R}^{T} \mathbf{R}=\right.$ $\left.\mathbf{I}_{n}, \operatorname{det}(\mathbf{R})=1\right\}$ indicates the special orthogonal group in dimension $n$. The linear span of vectors $\mathbf{x}_{1}, \mathbf{x}_{2}, \ldots, \mathbf{x}_{n}$ is denoted by $\operatorname{span}\left\{\mathbf{x}_{1}, \mathbf{x}_{2}, \ldots, \mathbf{x}_{n}\right\}$. Two vectors $\mathbf{x}, \mathbf{y} \in \mathbb{R}^{n}$ are orthogonal, written $\mathbf{x} \perp \mathbf{y}$, if $\mathbf{x}^{T} \mathbf{y}=0$. The orthogonal complement of $\mathcal{M} \subset \mathbb{R}^{n}$, a linear subspace, is defined as $\mathcal{M}^{\perp}=\left\{\mathbf{x} \in \mathbb{R}^{n}: \mathbf{x} \perp \mathbf{y}\right.$ for all $\left.\mathbf{y} \in \mathcal{M}\right\}$. The subspaces $\mathcal{M}, \mathcal{N} \subset \mathbb{R}^{n}$ are complementary if $\mathcal{M} \oplus \mathcal{N}=\mathbb{R}^{n}$, where $\oplus$ denotes the direct sum. $\mathbf{V} \in \mathbb{R}^{n \times m}$ and $\mathbf{W} \in \mathbb{R}^{n \times m}$ is a biorthogonal pair, if $\mathbf{W}^{T} \mathbf{V}=\mathbf{I}_{m}$.

\section{Preliminaries}

In this section, we briefly review the discrete-time consensus protocol and recall various definitions and results on projectors that will support our subsequent developments.

\section{A. Discrete-time consensus protocol}

Let $\mathcal{G}=(V, E)$ be an undirected graph, where $V=$ $\{1, \ldots, n\}$ is the set of nodes (or agents), and $E \subseteq V \times V$ is the set of edges. All $\mathcal{G}$ 's are assumed to be finite graphs with no self-loops or multiple edges [29].

\section{Definition 1 (Adjacency and Laplacian matrix)}

- The adjacency matrix $\mathbf{A}=\left[a_{i j}\right]$ of graph $\mathcal{G}$ is an $n \times n$ matrix defined as $a_{i j}=1$ if $\{i, j\} \in E$ and $a_{i j}=0$ otherwise.

- The Laplacian matrix of graph $\mathcal{G}$ is an $n \times n$ symmetric positive semidefinite matrix defined as $\mathbf{L}=\mathbf{D}-\mathbf{A}$ where $\mathbf{D}=\operatorname{diag}(\mathbf{A} \mathbb{1})$ is the degree matrix and $\mathbb{1}$ is a column vector of $n$ ones.

The discrete-time consensus protocol is defined as follows,

$$
\mathbf{x}(k+1)=\mathbf{P} \mathbf{x}(k), \quad k \in\{0,1,2, \ldots\},
$$

where $\mathbf{P}=\mathbf{I}_{n}-\varepsilon \mathbf{L}$ is the Perron matrix of $\mathcal{G}$ with parameter $\varepsilon, \mathbf{x}=\left[x_{1}, x_{2}, \ldots, x_{n}\right]^{T} \in \mathbb{R}^{n}$ is the state vector, 
and $0<\varepsilon<1 / d_{\max }(\mathcal{G})$ is the step-size, with $d_{\max }(\mathcal{G})=$ $\max (\mathbf{A} \mathbb{1})$ the maximum degree of $\mathcal{G}[1$, Sect. IIC]. System (1) is the one-step Euler discretization of the continuoustime Laplacian flow $\dot{\mathbf{x}}(t)=-\mathbf{L} \mathbf{x}(t)$. The Perron matrix is a symmetric, nonnegative matrix (i.e. all its entries are nonnegative). Moreover, it is row stochastic, i.e. it satisfies $\mathbf{P} \mathbb{1}=\mathbb{1}$, which means that $\mathbb{1}$ is the eigenvector of $\mathbf{P}$ associated with the eigenvalue 1 [3, Ch. 2]. It is well known that if the graph $\mathcal{G}$ is connected, then,

$$
\lim _{k \rightarrow \infty} \mathbf{x}(k)=\frac{1}{n} \mathbb{1} \mathbb{1}^{T} \mathbf{x}_{0},
$$

where $\mathbf{x}_{0} \triangleq\left[x_{1}(0), \ldots, x_{n}(0)\right]^{T}$ is the vector of initial conditions, i.e. average consensus is achieved. The convergence factor to average consensus is the second largest eigenvalue of $\mathbf{P}$ [3, Ch. 10].

\section{B. Orthogonal and oblique projections, reflections}

The following definitions and results are drawn from [27, Ch. 1-2], [28] and [30].

Definition 2 (Def. 2.1, [27]) A matrix $\Pi \in \mathbb{R}^{n \times n}$ is a projection (idempotent matrix), if and only if $\Pi^{2}=\Pi$. $\diamond$

Definition 3 (Def. 2.2, [27]) Let $\mathcal{M}, \mathcal{N} \subset \mathbb{R}^{n}$ be complementary subspaces. For each $\mathbf{z} \in \mathbb{R}^{n}$, there exists a unique decomposition of the form, $\mathbf{z}=\mathbf{x}+\mathbf{y}$ where $\mathbf{x} \in \mathcal{M}$, $\mathbf{y} \in \mathcal{N}$. The transformation $\mathbf{\Pi}_{\mathcal{M}, \mathcal{N}}$ defined by $\boldsymbol{\Pi}_{\mathcal{M}, \mathcal{N}} \mathbf{z} \triangleq \mathbf{x}$ is called projection onto $\mathcal{M}$ along $\mathcal{N}$. The transformation $\boldsymbol{\Pi}_{\mathcal{N}, \mathcal{M}}$ defined by $\boldsymbol{\Pi}_{\mathcal{N}, \mathcal{M}} \mathbf{z} \triangleq \mathbf{y}$ is called projection onto $\mathcal{N}$ along $\mathcal{M}$. The vector $\mathrm{x}$ is the projection of $\mathrm{z}$ onto $\mathcal{M}$ along $\mathcal{N}$, and $\mathbf{y}$ is the projection of $\mathbf{z}$ onto $\mathcal{N}$ along $\mathcal{M}$.

Definition 4 (Def. 2.3, [27]) The projection $\Pi$ onto $\mathcal{M}$ along $\mathcal{M}^{\perp}$ is called orthogonal.

Definition 5 (Def. 2.4, [27]) Let $\mathcal{M} \subset \mathbb{R}^{n}$ be a subspace. $\Pi \in \mathbb{R}^{n \times n}$ is the orthogonal projection onto $\mathcal{M}$ if (a) $\operatorname{Im}(\boldsymbol{\Pi})=\mathcal{M}$, (b) $\boldsymbol{\Pi}^{2}=\boldsymbol{\Pi}$, (c) $\boldsymbol{\Pi}^{T}=\boldsymbol{\Pi}$.

Projections that are not orthogonal projections, are called oblique projections.

Theorem 1 (Th. $2.8,[27]) \Pi$ is a projection if and only if $\mathbf{I}_{n}-\boldsymbol{\Pi}$ is also a projection. If $\boldsymbol{\Pi}$ is a projection onto $\mathcal{M}$ along $\mathcal{N}$, then $\mathbf{I}_{n}-\Pi$ is a projection onto $\mathcal{N}$ along $\mathcal{M}$. $\diamond$

It is also straightforward to show that $\Pi$ is a projection if and only if $\boldsymbol{\Pi}^{T}$ is also a projection.

Theorem 2 (Th. 2.10, [27] and p. 58, [28]) Any projection $\Pi \in \mathbb{R}^{n \times n}$ may have only 0 and 1 eigenvalues. The multiplicity of the eigenvalue 1 is $\operatorname{rank}(\boldsymbol{\Pi})=\operatorname{trace}(\boldsymbol{\Pi})$. $\diamond$

Theorem 3 (Th. 2.16, [27]) The linear transformation $\Pi$ is an orthogonal projection if and only if $\boldsymbol{\Pi}=\boldsymbol{\Pi}^{2}=\boldsymbol{\Pi}^{T}$. Moreover, the orthogonal projections are positive semidefinite and satisfy $\|\mathbf{\Pi} \mathbf{x}\|_{2} \leq\|\mathbf{x}\|_{2}$ for all $\mathbf{x} \in \mathbb{R}^{n}$.

Definition 6 (Def. 3.1.1, [30]) A matrix $\Psi \in \mathbb{R}^{n \times n}$ is a reflection if $\boldsymbol{\Psi}$ is symmetric and orthogonal.

Any reflection $\boldsymbol{\Psi}$ is an involutory matrix, i.e. $\boldsymbol{\Psi}^{2}=\mathbf{I}_{n}$, each of whose eigenvalues is 1 or -1 . The following proposition provides an interesting link between projections and reflections.

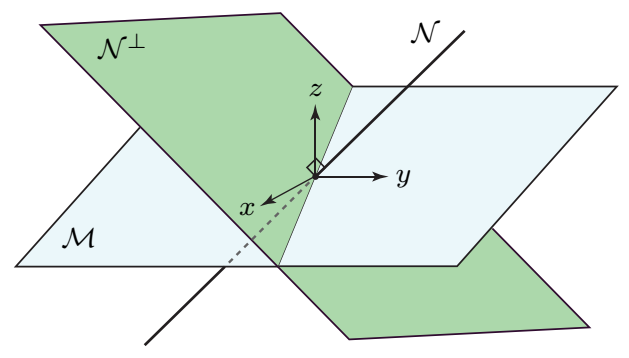

Fig. 1. Example of complementary subspaces $\mathcal{M}$ and $\mathcal{N}$ in $\mathbb{R}^{3}$.

Proposition 1 (Fact 3.14.1, [30]) If $\Pi \in \mathbb{R}^{n \times n}$ is an orthogonal projection, then $\boldsymbol{\Psi} \triangleq 2 \boldsymbol{\Pi}-\mathbf{I}_{n}$ is a reflection. Conversely, if $\boldsymbol{\Psi} \in \mathbb{R}^{n \times n}$ is a reflection, then $\boldsymbol{\Pi} \triangleq \frac{1}{2}\left(\boldsymbol{\Psi}+\mathbf{I}_{n}\right)$ is an orthogonal projection.

In fact, the affine mapping $\mathbf{f}(\boldsymbol{\Pi}) \triangleq 2 \boldsymbol{\Pi}-\mathbf{I}_{n}$ from orthogonal projections to reflections, is bijective. We also observe that if $\Pi_{\mathcal{M}}$ is the orthogonal projection onto $\mathcal{M}$, then $\left(2 \boldsymbol{\Pi}_{\mathcal{M}}-\mathbf{I}_{n}\right) \mathbf{z}$ is the reflection of $\mathbf{z} \in \mathbb{R}^{n}$ across $\mathcal{M}^{\perp}$.

We report below some representations of projections that will be used in Sect. III.

Theorem 4 (Th. 2.26, [27]) Let $\mathcal{M}, \mathcal{N} \subset \mathbb{R}^{n}$ be complementary subspaces, and let the columns of $\mathbf{V} \in \mathbb{R}^{n \times r}$ and $\mathbf{W} \in \mathbb{R}^{n \times r}$ form a basis for $\mathcal{M}$ and $\mathcal{N}^{\perp}$, respectively. Then, $\mathbf{\Pi}_{\mathcal{M}, \mathcal{N}}=\mathbf{V}\left(\mathbf{W}^{T} \mathbf{V}\right)^{-1} \mathbf{W}^{T}$.

Note that either $\mathbf{V}$ and $\mathbf{W}\left(\mathbf{W}^{T} \mathbf{V}\right)^{-T}$ or $\mathbf{V}\left(\mathbf{W}^{T} \mathbf{V}\right)^{-1}$ and $\mathbf{W}$ are biorthogonal pairs, and that $\left(\mathbf{W}^{T} \mathbf{V}\right)^{-1}$ can be interpreted as a "normalizing factor". In the 3D example of Fig. 1, we have:

$$
\mathbf{V}=\left[\begin{array}{ll}
1 & 0 \\
0 & 1 \\
0 & 0
\end{array}\right], \quad \mathbf{W}=\left[\begin{array}{rr}
1 & 0 \\
0 & 2 \\
0 & -2
\end{array}\right]
$$

being $\mathcal{N}=\operatorname{span}\left\{[0,1,1]^{T}\right\}$. Note that $\mathbf{V}$ and $\mathbf{W}$ is not a biorthogonal pair, since $\mathbf{W}^{T} \mathbf{V}=\operatorname{diag}(1,2)$.

Corollary 1 (Cor. 2.27, [27]) If the columns of $\mathbf{V}=$ $\left[\mathbf{v}_{1}, \mathbf{v}_{2}, \ldots, \mathbf{v}_{r}\right]$ form an orthonormal basis for a subspace $\mathcal{M} \subset \mathbb{R}^{n}$, then $\boldsymbol{\Pi}_{\mathcal{M}}=\mathbf{V V}^{T}$ is the unique orthogonal projection onto $\mathcal{M}$.

Lemma 1 (Lemma 2.30, [27]) Let $\mathcal{M}, \mathcal{N} \subset \mathbb{R}^{n}$ be complementary subspaces, and let $\mathbf{U}=\left[\mathbf{U}_{1}, \mathbf{U}_{2}\right]$ and $\mathbf{V}=$ $\left[\mathbf{V}_{1}, \mathbf{V}_{2}\right]$ be orthogonal matrices such that $\mathbf{U}_{1}$ and $\mathbf{U}_{2}$ are bases for $\mathcal{M}$ and $\mathcal{M}^{\perp}$, respectively, and $\mathbf{V}_{1}$ and $\mathbf{V}_{2}$ are bases for $\mathcal{N}$ and $\mathcal{N}^{\perp}$, respectively. Then,

$$
\boldsymbol{\Pi}_{\mathcal{M}, \mathcal{N}}=\mathbf{U}\left[\begin{array}{cc}
\mathbf{0} & \left(\mathbf{V}_{2}^{T} \mathbf{U}_{1}\right)^{-1} \\
\mathbf{0} & \mathbf{0}
\end{array}\right] \mathbf{V}^{T} .
$$

Matrices $\mathbf{U}$ and $\mathbf{V}$ can be chosen such that we obtain the Singular Value Decomposition (SVD) of $\Pi_{\mathcal{M}, \mathcal{N}}$.

Any projection is diagonalizable by a similarity transformation. In particular, the following result can be proved using Schur's theorem [27, Th. 1.28].

Theorem 5 (Th. 2.31, [27]) If $\Pi \in \mathbb{R}^{n \times n}$ is a projection of rank $m$, then there is an orthogonal matrix $\mathbf{V}$ such that,

$$
\mathbf{\Pi}=\mathbf{V}\left[\begin{array}{cc}
\mathbf{I}_{m} & \mathbf{B} \\
\mathbf{0} & \mathbf{0}_{n-m}
\end{array}\right] \mathbf{V}^{T},
$$

where matrix $\mathbf{B} \in \mathbb{R}^{m \times(n-m)}$ is arbitrary. 
We finally recall here some connections between projections and generalized inverses [28, Ch. 2]. Moore's original definition of Moore-Penrose inverse is the following:

Definition 7 (Def. 2.52, [27]) If $\mathbf{M} \in \mathbb{R}^{m \times n}$, then the generalized inverse of $\mathbf{M}$ is defined to be the unique matrix $\mathbf{M}^{+}$such that:

(a) $\mathrm{MM}^{+}=\Pi_{\operatorname{Im}(\mathbf{M})}$,

(b) $\mathbf{M}^{+} \mathbf{M}=\boldsymbol{\Pi}_{\operatorname{Im}\left(\mathbf{M}^{+}\right)}=\boldsymbol{\Pi}_{\operatorname{Im}\left(\mathbf{M}^{T}\right)}$.

The next theorem provides useful properties of the MoorePenrose inverse (cf. [27, Def. 1.54]).

Theorem 6 (Th. 1.60, [27]) Let $\mathbf{M}^{+} \in \mathbb{R}^{n \times m}$ be the Moore-Penrose inverse of $\mathbf{M} \in \mathbb{R}^{m \times n}$. Then,

(a) $\operatorname{Im}\left(\mathbf{M}^{+}\right)=\operatorname{Im}\left(\mathbf{M}^{T}\right)=\operatorname{Im}\left(\mathbf{M}^{+} \mathbf{M}\right)=\operatorname{Im}\left(\mathbf{M}^{T} \mathbf{M}\right)$,

(b) $\operatorname{Im}\left(\mathbf{I}_{n}-\mathbf{M}^{+} \mathbf{M}\right)=\operatorname{ker}\left(\mathbf{M}^{+} \mathbf{M}\right)=\operatorname{ker}(\mathbf{M})=$ $\operatorname{Im}\left(\mathbf{M}^{T}\right)^{\perp}$.

\section{STATE-CONSTRAINED CONSENSUS PROTOCOLS}

In this section, we present the main theoretical results of this paper. In what follows, we make the standing assumption that the graph $\mathcal{G}$ is connected.

Theorem 7 (Orthogonal projection) Let us suppose that the following constraint is imposed on the state vector of consensus protocol (1),

$$
\mathbf{x}(k+1) \in \mathcal{M} \subset \mathbb{R}^{n}, \quad k \in\{0,1,2, \ldots\},
$$

where the columns of $\mathbf{V}=\left[\mathbf{v}_{1}, \mathbf{v}_{2}, \ldots, \mathbf{v}_{r}\right] \in \mathbb{R}^{n \times r}$ form an orthonormal basis for the linear subspace $\mathcal{M}$ and $\mathbb{1} \in \operatorname{span}\left\{\mathbf{v}_{1}, \mathbf{v}_{2}, \ldots, \mathbf{v}_{r}\right\}$. Consider the projected consensus protocol,

$$
\mathbf{x}_{\mathrm{or}}(k+1)=\boldsymbol{\Pi}_{\mathcal{M}} \mathbf{P} \mathbf{x}_{\mathrm{or}}(k), \quad \mathbf{x}_{\mathrm{or}}(0)=\mathbf{x}_{0},
$$

where $\boldsymbol{\Pi}_{\mathcal{M}}=\mathbf{V} \mathbf{V}^{T} \in \mathbb{R}^{n \times n}$ is the orthogonal projection onto $\mathcal{M}$ (cf. Corollary 1), and let us assume that

$$
\operatorname{rank}\left(\mathbf{I}_{n}-\boldsymbol{\Pi}_{\mathcal{M}} \mathbf{P}\right)=n-1
$$

Then, the solution to (3) satisfies,

$$
\lim _{k \rightarrow \infty} \mathbf{x}_{\text {or }}(k)=\frac{1}{n} \mathbb{1}^{T} \mathbf{x}_{0} .
$$

Proof: To prove (5), we need to study the spectrum of the dynamics matrix $\boldsymbol{\Pi}_{\mathcal{M}} \mathbf{P}$ of system (3). Note that while $\boldsymbol{\Pi}_{\mathcal{M}}$ and $\mathbf{P}$ are both symmetric matrices, their product $\Pi_{\mathcal{M}} \mathbf{P}$, is neither a symmetric nor a row-stochastic matrix, in general. Without loss of generality, the orthonormal basis for $\mathcal{M}$ can be written as $\mathbf{V}=[\mathbb{1} / \sqrt{n}, \overline{\mathbf{V}}], \overline{\mathbf{V}}=\left[\mathbf{v}_{2}, \ldots, \mathbf{v}_{r}\right] \in$ $\mathbb{R}^{n \times(r-1)}$. Therefore,

$$
\mathbf{V}^{T} \mathbf{V}=\left[\begin{array}{cc}
1 & \frac{1}{\sqrt{n}} \mathbb{1}^{T} \overline{\mathbf{V}} \\
\frac{1}{\sqrt{n}} \overline{\mathbf{V}}^{T} \mathbb{1} & \overline{\mathbf{V}}^{T} \overline{\mathbf{V}}
\end{array}\right]=\mathbf{I}_{r},
$$

which implies that $\overline{\mathbf{V}}^{T} \mathbb{1}=\mathbf{0}$. Then,

$$
\mathbf{\Pi}_{\mathcal{M}} \mathbf{P} \mathbb{1}=\boldsymbol{\Pi}_{\mathcal{M}} \mathbb{1}=\mathbf{V} \mathbf{V}^{T} \mathbb{1}=\left(\frac{1}{n} \mathbb{1} \mathbb{1}^{T}+\overline{\mathbf{V}} \overline{\mathbf{V}}^{T}\right) \mathbb{1}=\mathbb{1} .
$$

Hence, each of the rows of $\Pi_{\mathcal{M}} \mathbf{P}$ has unit sum, and 1 is an eigenvalue of $\Pi_{\mathcal{M}} \mathbf{P}$ with eigenvector $\mathbb{1}$. The rank condition (4) guarantees that the geometric multiplicity of this eigenvalue is 1 . Note that since $\mathbf{P}$ is full rank, then
$\operatorname{rank}\left(\boldsymbol{\Pi}_{\mathcal{M}} \mathbf{P}\right)=\operatorname{rank}\left(\boldsymbol{\Pi}_{\mathcal{M}}\right)=\operatorname{rank}\left(\mathbf{V V}^{T}\right)=\operatorname{rank}(\mathbf{V})=r$. Therefore, $\Pi_{\mathcal{M}} \mathbf{P}$ has $n-r$ zero eigenvalues. Let us now focus on the nonzero eigenvalues of $\Pi_{\mathcal{M}} \mathbf{P}$. Taking inspiration from the proof of Lemma 2.47 in [27], we have that the equality,

$$
\boldsymbol{\Pi}_{\mathcal{M}} \mathbf{P} \mathbf{u}=\lambda \mathbf{u}
$$

where $\lambda$ is the eigenvalue of $\Pi_{\mathcal{M}} \mathbf{P}$ with corresponding eigenvector $\mathbf{u}$, implies $\Pi_{\mathcal{M}}^{2} \mathbf{P} \mathbf{u}=\lambda \Pi_{\mathcal{M}} \mathbf{u}$. But since $\boldsymbol{\Pi}_{\mathcal{M}}^{2}=\boldsymbol{\Pi}_{\mathcal{M}}$, then $\boldsymbol{\Pi}_{\mathcal{M}} \mathbf{P} \mathbf{u}=\lambda \boldsymbol{\Pi}_{\mathcal{M}} \mathbf{u}$. By comparing the last equation with (6), we deduce that $\mathbf{u}=\Pi_{\mathcal{M}} \mathbf{u}$. As a consequence, since $\Pi_{\mathcal{M}}=\Pi_{\mathcal{M}}^{T}$,

$\mathbf{u}^{H} \boldsymbol{\Pi}_{\mathcal{M}} \mathbf{P} \mathbf{u}=\left(\boldsymbol{\Pi}_{\mathcal{M}}^{H} \mathbf{u}\right)^{H} \mathbf{P} \mathbf{u}=\left(\boldsymbol{\Pi}_{\mathcal{M}} \mathbf{u}\right)^{H} \mathbf{P} \mathbf{u}=\mathbf{u}^{H} \mathbf{P} \mathbf{u}$.

But from (6), we also have,

$$
\mathbf{u}^{H} \boldsymbol{\Pi}_{\mathcal{M}} \mathbf{P} \mathbf{u}=\lambda \mathbf{u}^{H} \mathbf{u}
$$

By comparing (7) with (8), and by recalling Rayleigh-Ritz's theorem [27, Th. 1.81], we find that,

$$
\lambda=\frac{\mathbf{u}^{H} \mathbf{P} \mathbf{u}}{\mathbf{u}^{H} \mathbf{u}} \in\left[\lambda_{\min }(\mathbf{P}), \lambda_{\max }(\mathbf{P})\right],
$$

where $\lambda_{\min }(\mathbf{P})$ and $\lambda_{\max }(\mathbf{P})$ denote the minimum and maximum eigenvalue of $\mathbf{P}$. In summary, we have proved that $\boldsymbol{\Pi}_{\mathcal{M}} \mathbf{P}$ has $n-r$ zero eigenvalues, that the nonzero eigenvalues are real and take values in $\left[\lambda_{\min }(\mathbf{P}), 1\right]$, and that $\mathbb{1}$ is the eigenvector associated with the eigenvalue 1 , from which (5) directly follows.

Note that (3) simply proceeds by projecting each step of consensus protocol (1) onto the constraint subspace $\mathcal{M}$, thus ensuring positive invariance [31]. The orthogonal projection $\boldsymbol{\Pi}_{\mathcal{M}}$ is instrumental in enforcing constraint (2), but it does modify the spectrum of $\mathbf{P}$ and affects the convergence rate to average consensus. Nevertheless, the sequences $\left\{\mathbf{x}_{\mathrm{or}}(k)\right\}$ and $\{\mathbf{x}(k)\}$ converge to the same consensus value.

Remark 1 (Nonnegative projections) If $\Pi_{\mathcal{M}}$ is nonnegative, then $\Pi_{\mathcal{M}} \mathbf{P}$ is nonnegative as well. Since the row-sums of $\Pi_{\mathcal{M}} \mathbf{P}$ are all equal to 1 , then $\Pi_{\mathcal{M}} \mathbf{P}$ is row-stochastic and the statement of Theorem 7 becomes a direct consequence of [3, Th. 5.1]. For further results on nonnegative projections, the reader is referred to [27, pp. 48-50]. $\diamond$

The first of the next two corollaries states that Theorem 7 is still valid when the orthogonal projection $\boldsymbol{\Pi}_{\mathcal{M}}$ is replaced with the reflection $\Psi_{\mathcal{M}}=2 \Pi_{\mathcal{M}}-\mathbf{I}_{n}$ (recall Proposition 1), while the second one specializes it to linear state equality constraints.

Corollary 2 (Reflection) Let us suppose that the assumptions of Theorem 7 hold true. Then, provided that $\operatorname{rank}\left(\mathbf{I}_{n}-\right.$ $\left.\Psi_{\mathcal{M}} \mathbf{P}\right)=n-1$, the solution to the reflected consensus protocol,

$$
\mathbf{x}_{\mathrm{re}}(k+1)=\Psi_{\mathcal{M}} \mathbf{P} \mathbf{x}_{\mathrm{re}}(k), \quad \mathbf{x}_{\mathrm{re}}(0)=\mathbf{x}_{0},
$$

satisfies $\lim _{k \rightarrow \infty} \mathbf{x}_{\mathrm{re}}(k)=\frac{1}{n} \mathbb{1 1}^{T} \mathbf{x}_{0}$ where $\boldsymbol{\Psi}_{\mathcal{M}}=2 \boldsymbol{\Pi}_{\mathcal{M}}$ $\mathbf{I}_{n}$ is the reflection across $\mathcal{M}^{\perp}$.

Proof: Due to space limitations, the proof is omitted. It is reported in [32], the extended version of this article.

Note that unlike Theorem 7, in this case the state vector $\mathrm{x}_{\mathrm{re}}$ of system (9) "bounces off" the subspace $\mathcal{M}^{\perp}$. 
Corollary 3 (Orthogonal projection: linear equality constraints) Let us suppose that the following equality constraint is imposed on the state vector of protocol (1),

$$
\mathbf{M x}(k+1)=\mathbf{0}, \quad k \in\{0,1,2, \ldots\},
$$

where matrix $\mathbf{M} \in \mathbb{R}^{q \times n}$ has row rank $m \leq q$ and satisfies $\mathbf{M} \mathbb{1}=\mathbf{0}$. Consider the projected consensus protocol,

$$
\mathbf{x}_{\text {or }}(k+1)=\Pi_{\text {ker }(\mathbf{M})} \mathbf{P} \mathbf{x}_{\text {or }}(k), \quad \mathbf{x}_{\text {or }}(0)=\mathbf{x}_{0},
$$

where $\mathbf{\Pi}_{\mathrm{ker}(\mathbf{M})}=\mathbf{V V}^{T}$ is the orthogonal projection onto the null space of $\mathbf{M}$, i.e. the columns of $\mathbf{V} \in \mathbb{R}^{n \times(n-m)}$ form a basis which satisfies $\mathbf{M V}=\mathbf{0}$ and $\mathbf{V}^{T} \mathbf{V}=\mathbf{I}_{n-m}$. Then, under condition (4), the state trajectories of protocol (11) satisfy (5).

Proof: Since $\mathbf{M} \mathbb{1}=\mathbf{0}$, then $\mathbb{1} \in \operatorname{ker}(\mathbf{M})$ and we can write the orthonormal basis of $\operatorname{ker}(\mathbf{M})$ as $\mathbf{V}=[\mathbb{1} / \sqrt{n}, \overline{\mathbf{V}}]$ where $\overline{\mathbf{V}} \in \mathbb{R}^{n \times(n-m-1)}$. The rest of the proof is an immediate transcription of that of Theorem 7.

As illustrated in Sect. IV, matrix $\mathbf{M}$ can be used to encode a variety of constraints frequently encountered in distributed robotics (e.g. collision avoidance or connectivity maintenance constraints). Note that $\Pi_{k e r(M)}$ can be readily found by computing the SVD of $\mathbf{M}$. In fact, if $\mathbf{M}$ is decomposed as $\mathbf{M}=\left[\mathbf{U}_{m}, \overline{\mathbf{U}}_{m}\right] \boldsymbol{\Sigma}\left[\mathbf{V}_{m}, \overline{\mathbf{V}}_{m}\right]^{T}$ where $\left[\mathbf{U}_{m}, \overline{\mathbf{U}}_{m}\right],\left[\mathbf{V}_{m}, \overline{\mathbf{V}}_{m}\right]$ are orthogonal matrices with $\mathbf{U}_{m} \in \mathbb{R}^{q \times m}, \overline{\mathbf{U}}_{m} \in \mathbb{R}^{q \times(q-m)}, \mathbf{V}_{m} \in \mathbb{R}^{n \times m}, \overline{\mathbf{V}}_{m} \in$ $\mathbb{R}^{n \times(n-m)}$, and $\boldsymbol{\Sigma} \in \mathbb{R}^{q \times n}$ is a rectangular diagonal matrix with nonnegative real numbers in decreasing order on the diagonal, then $\overline{\mathbf{V}}_{m} \overline{\mathbf{V}}_{m}^{T}$ is the orthogonal projection onto $\operatorname{ker}(\mathbf{M})$ [27, Th. 2.36]. Moreover, $\mathbf{V}_{m} \mathbf{V}_{m}^{T}, \mathbf{U}_{m} \mathbf{U}_{m}^{T}$ and $\overline{\mathbf{U}}_{m} \overline{\mathbf{U}}_{m}^{T}$ are the orthogonal projections onto $\operatorname{ker}(\mathbf{M})^{\perp}$, $\operatorname{Im}(\mathbf{M})$ and $\operatorname{Im}(\mathbf{M})^{\perp}$, respectively.

The next theorem extends Theorem 7 to the more challenging case of oblique projections (recall Theorem 4).

Theorem 8 (Oblique projection) Let us suppose that constraint (2) is imposed on the state vector of protocol (1). Let $\mathcal{M}, \mathcal{N} \subset \mathbb{R}^{n}$ be complementary subspaces. Let the columns of $\mathbf{V}=\left[\mathbf{v}_{1}, \mathbf{v}_{2}, \ldots, \mathbf{v}_{r}\right] \in \mathbb{R}^{n \times r}$ and $\mathbf{W}=$ $\left[\mathbf{w}_{1}, \mathbf{w}_{2}, \ldots, \mathbf{w}_{r}\right] \in \mathbb{R}^{n \times r}$ form a basis for $\mathcal{M}$ and $\mathcal{N}^{\perp}$, respectively, and let $\mathbf{V}$ and $\mathbf{W}$ be a biorthogonal pair, i.e. $\mathbf{W}^{T} \mathbf{V}=\mathbf{I}_{r}$. Let $\mathbb{1} \in \operatorname{span}\left\{\mathbf{v}_{1}, \mathbf{v}_{2}, \ldots, \mathbf{v}_{r}\right\} \cap$ $\operatorname{span}\left\{\mathbf{w}_{1}, \mathbf{w}_{2}, \ldots, \mathbf{w}_{r}\right\}$. Consider the projected consensus protocol,

$$
\mathbf{x}_{\mathrm{ob}}(k+1)=\boldsymbol{\Pi}_{\mathcal{M}, \mathcal{N}} \mathbf{P} \mathbf{x}_{\mathrm{ob}}(k), \quad \mathbf{x}_{\mathrm{ob}}(0)=\mathbf{x}_{0},
$$

where $\boldsymbol{\Pi}_{\mathcal{M}, \mathcal{N}}=\mathbf{V W}^{T}$ and assume that $\operatorname{rank}\left(\mathbf{I}_{n}-\right.$ $\left.\Pi_{\mathcal{M}, \mathcal{N}} \mathbf{P}\right)=n-1$. Then, the solution to (12) satisfies,

$$
\lim _{k \rightarrow \infty} \mathbf{x}_{\mathrm{ob}}(k)=\frac{1}{n} \mathbb{1 1}^{T} \mathbf{x}_{0} .
$$

Proof: It is available in [32].

If $\mathbb{1} \notin \operatorname{span}\left\{\mathbf{w}_{1}, \mathbf{w}_{2}, \ldots, \mathbf{w}_{r}\right\}$, system (12) may still achieve consensus asymptotically, but not average consensus.

Remark 2 (On the distributed nature of the projected consensus protocols) Note that matrix $\Pi_{\mathcal{M}, \mathcal{N}} \mathbf{P}$ is less sparse than $\mathbf{P}$, in general, and it can be associated with a communication graph $\mathcal{G}^{\prime}=\left(V, E^{\prime}\right)$ with $E \subseteq E^{\prime}$, over which the dynamics of (12) evolve (cf. Fig. 4). This is not surprising, since to fulfill the state constraint (2) while still achieving average consensus, the communication between the robots must be more efficient. If the subspaces $\mathcal{M}$ and $\mathcal{N}$ are known a priori, protocol (12) (as well as protocol (3)) admits a distributed implementation over $\mathcal{G}^{\prime}$.

In the next theorem, we leverage Theorems 1 and 6 , and consider a special oblique projection for the case of linear state equality constraints. The "obliquity" of the projection hinges on the positive definite weight matrix $\Omega$, which alters the group decision value of protocol (1).

Theorem 9 (Weighted oblique projection: linear equality constraints) Let us suppose that constraint (10) is imposed on the state vector of protocol (1), where $\mathbf{M} \in \mathbb{R}^{q \times n}$ has full row rank and satisfies $\mathbf{M} \mathbb{1}=\mathbf{0}$. Consider the projected consensus protocol,

$$
\mathbf{x}_{\mathrm{wob}}(k+1)=\boldsymbol{\Pi}_{\mathrm{ker}(\mathbf{M})}^{\mathrm{ob}} \mathbf{P} \mathbf{x}_{\mathrm{wob}}(k), \quad \mathbf{x}_{\mathrm{wob}}(0)=\mathbf{x}_{0},
$$

where $\mathbf{\Pi}_{\mathrm{ker}(\mathbf{M})}^{\mathrm{ob}}=\mathbf{I}_{n}-\mathbf{M}_{R}^{\dagger} \mathbf{M}$ is the oblique projection onto the null space of $\mathbf{M}$ and $\mathbf{M}_{R}^{\dagger}=\boldsymbol{\Omega}^{-1} \mathbf{M}^{T}\left(\mathbf{M} \boldsymbol{\Omega}^{-1} \mathbf{M}^{T}\right)^{-1}$ is the weighted right pseudo-inverse of $\mathbf{M}$ [33, Sect. A.7], where $\Omega \in \mathbb{R}^{n \times n}$ is a positive definite matrix. Provided that $\operatorname{rank}\left(\mathbf{I}_{n}-\mathbf{\Pi}_{\mathrm{ker}(\mathbf{M})}^{\mathrm{ob}} \mathbf{P}\right)=n-1$, the state trajectories of (13) satisfy,

$$
\lim _{k \rightarrow \infty} \mathbf{x}_{\mathrm{wob}}(k)=\left(\boldsymbol{\mu}^{T} \mathbf{x}_{0}\right) \mathbb{1},
$$

where $\boldsymbol{\mu}$ is the left eigenvector of $\boldsymbol{\Pi}_{\mathrm{ker}(\mathbf{M})}^{\mathrm{ob}} \mathbf{P}$ with eigenvalue 1 , normalized to have unit sum, i.e. $\mathbb{1}^{T} \boldsymbol{\mu}=1$.

Proof: It is available in [32].

Finally, with reference to Theorem 7 , in the following proposition we study the effect of a rotation matrix $\mathbf{R}$ on the basis vectors of the constraint subspace $\mathcal{M}$.

Proposition 2 (Orthogonal similarity transformation of an orthogonal projection) Let the columns of $\mathbf{V}=\left[\mathbf{v}_{1}, \mathbf{v}_{2}, \ldots, \mathbf{v}_{r}\right] \in \mathbb{R}^{n \times r}$ form an orthonormal basis for the subspace $\mathcal{M}$, and let $\Pi_{\mathcal{M}}$ the orthogonal projection onto $\mathcal{M}$. Let $\mathcal{M}^{\mathbf{R}}$ be the subspace with orthonormal basis $\mathbf{R V}$, where $\mathbf{R} \in \operatorname{SO}(n)$. Then, $\mathbf{\Pi}_{\mathcal{M}}^{\mathbf{R}}=\mathbf{R} \boldsymbol{\Pi}_{\mathcal{M}} \mathbf{R}^{T}$ is the orthogonal projection onto $\mathcal{M}^{\mathbf{R}}$, and $\Pi_{\mathcal{M}}^{\mathbf{R}}$ and $\Pi_{\mathcal{M}}$ have the same spectrum.

Proof: We have that,

$\left(\boldsymbol{\Pi}_{\mathcal{M}}^{\mathbf{R}}\right)^{2}=\left(\mathbf{R} \boldsymbol{\Pi}_{\mathcal{M}} \mathbf{R}^{T}\right)\left(\mathbf{R} \boldsymbol{\Pi}_{\mathcal{M}} \mathbf{R}^{T}\right)=\mathbf{R} \boldsymbol{\Pi}_{\mathcal{M}}^{2} \mathbf{R}^{T}=\boldsymbol{\Pi}_{\mathcal{M}}^{\mathbf{R}}$, and $\left(\boldsymbol{\Pi}_{\mathcal{M}}^{\mathbf{R}}\right)^{T}=\left(\mathbf{R} \boldsymbol{\Pi}_{\mathcal{M}} \mathbf{R}^{T}\right)^{T}=\mathbf{R} \boldsymbol{\Pi}_{\mathcal{M}}^{T} \mathbf{R}^{T}=\boldsymbol{\Pi}_{\mathcal{M}}^{\mathbf{R}}$, hence owing to Definition $5, \Pi_{\mathcal{M}}^{\mathbf{R}}$ is the orthogonal projection onto $\mathcal{M}^{\mathbf{R}}$. Finally, since $\mathbf{R}^{T}=\mathbf{R}^{-1}$, matrices $\Pi_{\mathcal{M}}^{\mathbf{R}}$ and $\Pi_{\mathcal{M}}$ are similar and they have the same spectrum.

Note that the result of Proposition 2 can be extended to oblique projections, i.e. if we consider $\Pi_{\mathcal{M}, \mathcal{N}}^{\mathbf{R}}=$ $\mathbf{R} \Pi_{\mathcal{M}, \mathcal{N}} \mathbf{R}^{T}$ (cf. [27, Th. 2.35]).

\section{NUMERICAL EXPERIMENTS}

In this section, the theory presented in Sect. III is illustrated via numerical experiments. For its simplicity, the 2D rendezvous problem for single-integrator robots [5], is chosen here as a testing ground for the proposed projected consensus protocols. To improve readability, the discretetime state trajectories will be displayed as continuous curves. 

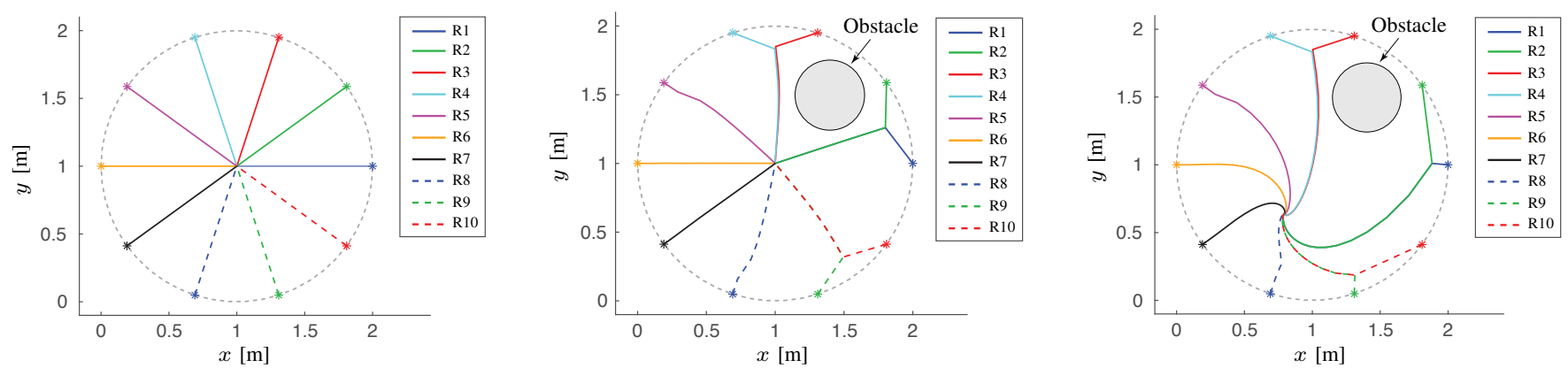

(a)
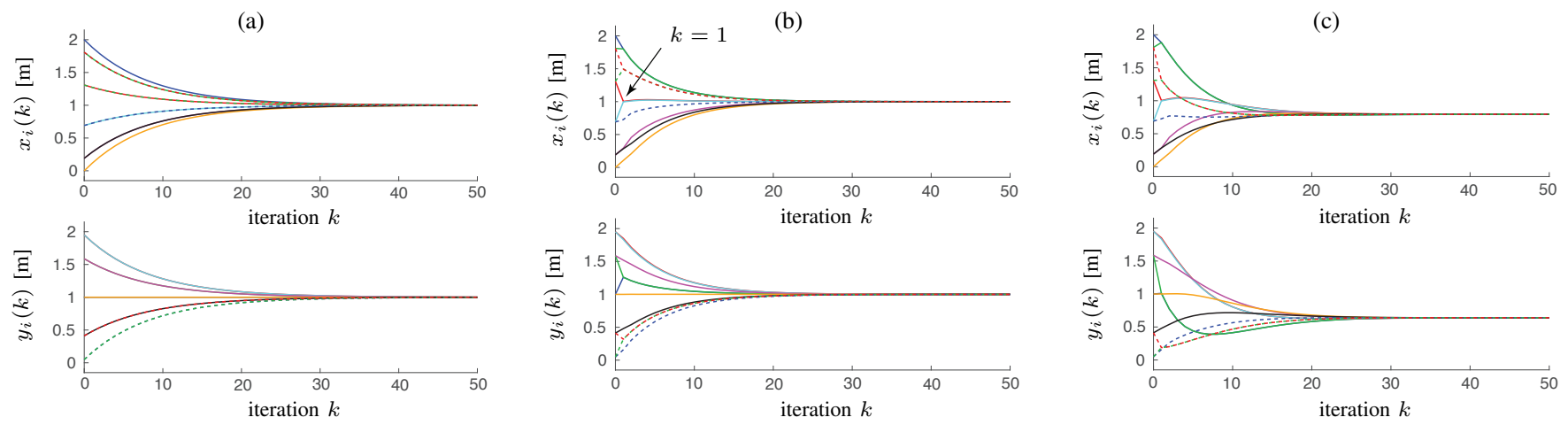

(d)

(e)

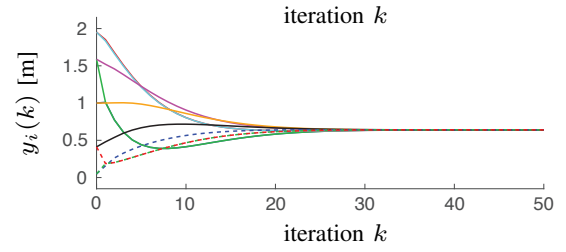

(f)

Fig. 2. Trajectory of the 10 robots (1st row), and corresponding time-evolution of their $x$ - and $y$-coordinates (2nd row). In the two rows, the same color convention is used. (a),(d) Unconstrained consensus protocol with communication graph $\mathcal{G}=C_{10}$ and step-size $\varepsilon=0.3$; (b),(e) Constrained consensus protocol, orthogonal projection onto $\operatorname{ker}\left(\mathbf{M}_{1}\right)$; (c),(f) Constrained consensus protocol, weighted oblique projection onto $\operatorname{ker}\left(\mathbf{M}_{1}\right)$.

The $n=10$ robots are initially arranged at equal intervals around a unit circle centered at point $(1,1)$ (dashed gray in Figs. 2(a)-(c) and 3(a)-(c)), i.e. $\mathbf{x}_{0}=[\cos (0), \sin (0), \cos (\pi / 5), \sin (\pi / 5), \ldots, \cos (9 \pi / 5)$, $\sin (9 \pi / 5)]^{T}+[1,1, \ldots, 1]^{T} \in \mathbb{R}^{20}$, and they have a prior knowledge of the surrounding environment.

Fig. 2(a) shows the trajectory of the robots obtained with the unconstrained consensus protocol,

$$
\mathbf{x}(k+1)=\left(\mathbf{P} \otimes \mathbf{I}_{2}\right) \mathbf{x}(k), \mathbf{x}(0)=\mathbf{x}_{0},
$$

with a cycle communication graph $\mathcal{G}=C_{10}$ and a stepsize $\varepsilon=0.3<1 / d_{\max }\left(C_{10}\right)=1 / 2$. The corresponding time-evolution of the $x$ - and $y$-coordinates of the robots for the first half of the 100-iteration simulation, is reported in Fig. 2(d) (the same color convention as in Fig. 2(a) is adopted here). As expected, the robots meet at point $(1,1)$. For comparison, Fig. 2(b) shows the trajectory of the robots generated by the constrained consensus protocol with orthogonal projection (cf. Corollary 3),

$$
\mathbf{x}_{\mathrm{or}}(k+1)=\left(\boldsymbol{\Pi}_{\mathcal{M}} \mathbf{P} \otimes \mathbf{I}_{2}\right) \mathbf{x}_{\mathrm{or}}(k), \quad \mathbf{x}_{\mathrm{or}}(0)=\mathbf{x}_{0},
$$

where $\mathcal{M}=\operatorname{ker}\left(\mathbf{M}_{1}\right) \subset \mathbb{R}^{10}$, with

$$
\mathbf{M}_{1}=\left[\begin{array}{rrrrrrrrrr}
-1 & 1 & 0 & 0 & 0 & 0 & 0 & 0 & 0 & 0 \\
0 & 0 & -10 & 10 & \frac{1}{3} & -\frac{1}{3} & 0 & 0 & 0 & 0 \\
0 & 0 & 0 & 0 & 0 & 0 & 0 & 0 & -\frac{1}{2} & \frac{1}{2}
\end{array}\right] .
$$

Matrix $\mathbf{M}_{1}$ allows here to encode "attraction forces" of various magnitude between pairs of robots. From Fig. 2(b) and Fig. 2(e), which reports the corresponding time-evolution of the $x$ - and $y$-coordinates of the robots, we observe that the trajectories of robots 1 and 2, 3 and 4, and 9 and 10, merge after one iteration (i.e. for $k=1$ ), but that the rendezvous point $(1,1)$ is unaffected. Robots 2 and 3 thus avoid the collision with the circular obstacle with radius $r=0.25 \mathrm{~m}$, centered at $(1.45,1.5)$, shown in Fig. 2(b) (shaded).

Fig. 2(c) reports the trajectory of the robots generated by the constrained consensus protocol with weighted oblique projection (cf. Theorem 9),

$\mathbf{x}_{\mathrm{wob}}(k+1)=\left(\boldsymbol{\Pi}_{\mathrm{ker}\left(\mathbf{M}_{1}\right)}^{\mathrm{ob}} \mathbf{P} \otimes \mathbf{I}_{2}\right) \mathbf{x}_{\mathrm{wob}}(k), \quad \mathbf{x}_{\mathrm{wob}}(0)=\mathbf{x}_{0}$,

where the diagonal weight matrix $\Omega=\operatorname{diag}(50,1,1,1,50$, $1,1,1,10,1)$, and Fig. 2(f) shows the time-evolution of the positions of the robots. In this case, the rendezvous point is $(0.79486,0.64031)$, cf. equation (14).

In Figs. 3(a),(d) the case of a general oblique projection,

$$
\mathbf{x}_{\mathrm{ob}}(k+1)=\left(\boldsymbol{\Pi}_{\mathcal{M}, \mathcal{N}} \mathbf{P} \otimes \mathbf{I}_{2}\right) \mathbf{x}_{\mathrm{ob}}(k), \quad \mathbf{x}_{\mathrm{ob}}(0)=\mathbf{x}_{0},
$$

is considered (cf. Theorem 8). Here, the nine columns of

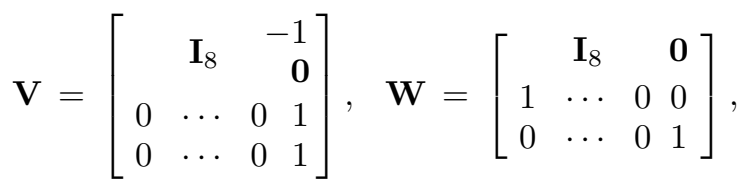

form a basis for the subspaces $\mathcal{M}$ and $\mathcal{N}^{\perp}$, respectively, being $\mathcal{N}=\operatorname{span}\left([1,0, \ldots, 0,-1,0]^{T}\right)$. The matrices $\mathbf{V}$ and $\mathbf{W}$ satisfy the conditions of Theorem 8, and as shown in Fig. 3(a), the subspace constraint $\mathcal{M}$ primarily affects the behavior of robots 1 and 9 . However, again, the rendezvous 


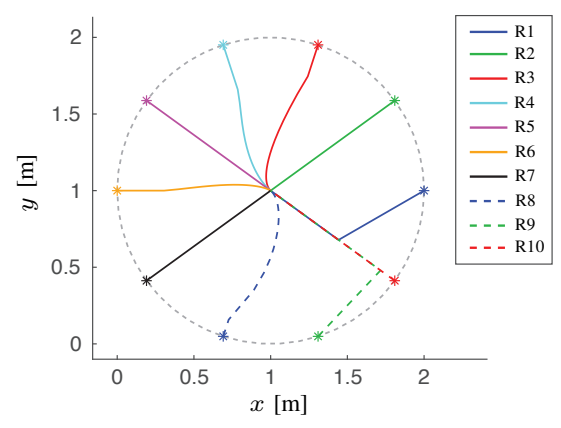

(a)
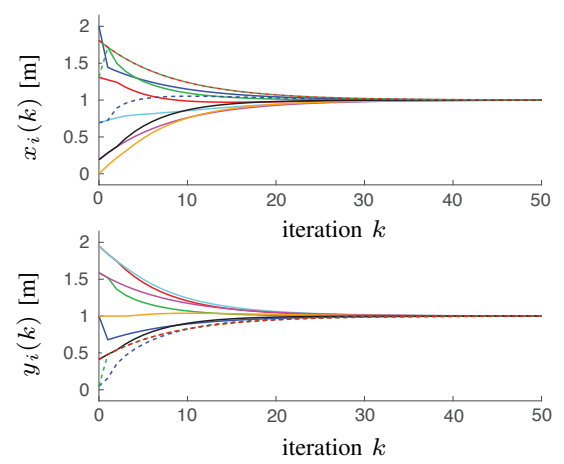

(d)

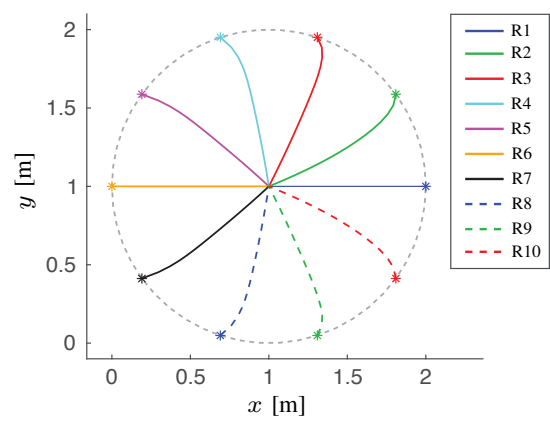

(b)
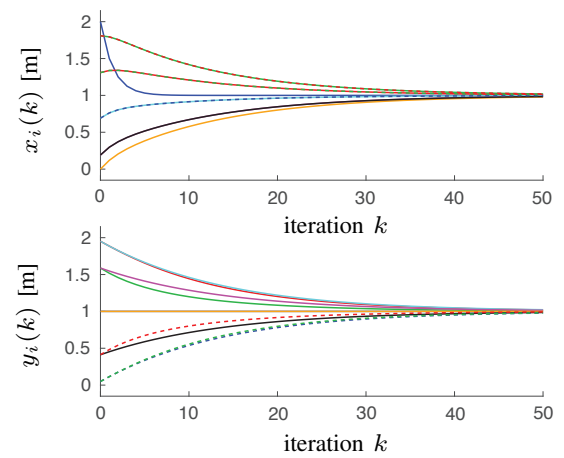

(e)

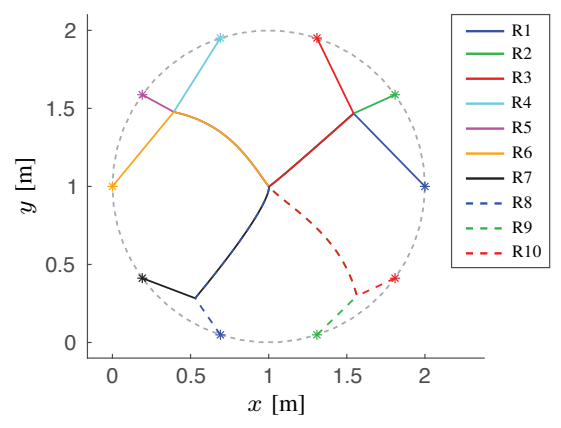

(c)
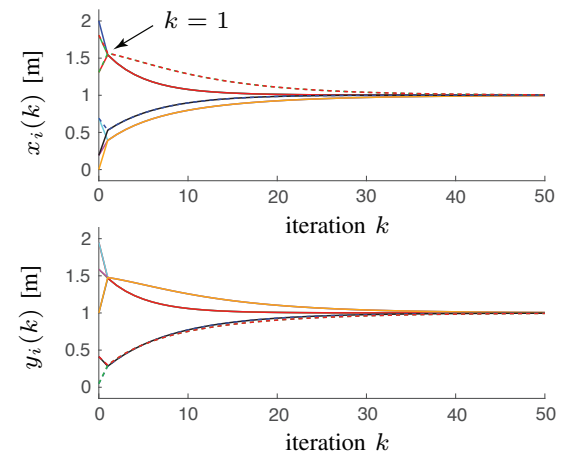

(f)

Fig. 3. Trajectory of the 10 robots (1st row), and corresponding time-evolution of their $x$ - and $y$-coordinates (2nd row). In the two rows, the same color convention is used. (a),(d) Constrained consensus protocol, general oblique projection $\Pi_{\mathcal{M}, \mathcal{N}}$; (b),(e) Unconstrained consensus protocol with communication graph $\mathcal{G}=W_{10}$ and step-size $\varepsilon=1 / 20$; (c),(f) Constrained consensus protocol, orthogonal projection onto $\operatorname{Im}\left(\mathbf{M}_{2}^{T}\right)$.

point is left unchanged, showing the convergence-preserving nature of protocol (17).

The trajectory of the robots obtained with protocol (15) using a wheel graph $\mathcal{G}=W_{10}$ and a step-size $\varepsilon=1 / 20<$ $1 / d_{\max }\left(W_{10}\right)=1 / 9$ is reported in Fig. 3(b). To draw a comparison, Fig. 3(c) shows the constrained trajectories of system (16) for $\mathcal{M}=\operatorname{Im}\left(\mathbf{M}_{2}^{T}\right) \subset \mathbb{R}^{10}$, where

$$
\mathbf{M}_{2}=\left[\begin{array}{cccccccccc}
\frac{1}{3} & \frac{1}{3} & \frac{1}{3} & 0 & 0 & 0 & 0 & 0 & 0 & 0 \\
0 & 0 & 0 & 0 & 0 & 0 & 2 & 2 & 0 & 0 \\
0 & 0 & 0 & 1 & 1 & 1 & 0 & 0 & 0 & 0 \\
0 & 0 & 0 & 0 & 0 & 0 & 0 & 0 & \frac{1}{2} & \frac{1}{2}
\end{array}\right] .
$$

Similarly to $\mathbf{M}_{1}$, matrix $\mathbf{M}_{2}$ is used here for coupling the motion of two pairs and two triplets of robots. In Figs. 3(c),(f), we see that to satisfy the imposed subspace constraint, four robot clusters emerge after one iteration, but that the rendezvous point remains $(1,1)$.

Finally, to get a sense of the amount of communication between the robots required by the six protocols studied in this section, Fig. 4 reports the sparsity pattern of the corresponding dynamics matrices (grayscale map of matrix entries).

\section{CONCLUSION AND FUTURE WORK}

In this paper, we leveraged subspace projectors to design a new class of state-constrained consensus protocols. The group decision properties of several variants of a prototypal algorithm have been studied, and numerical experiments
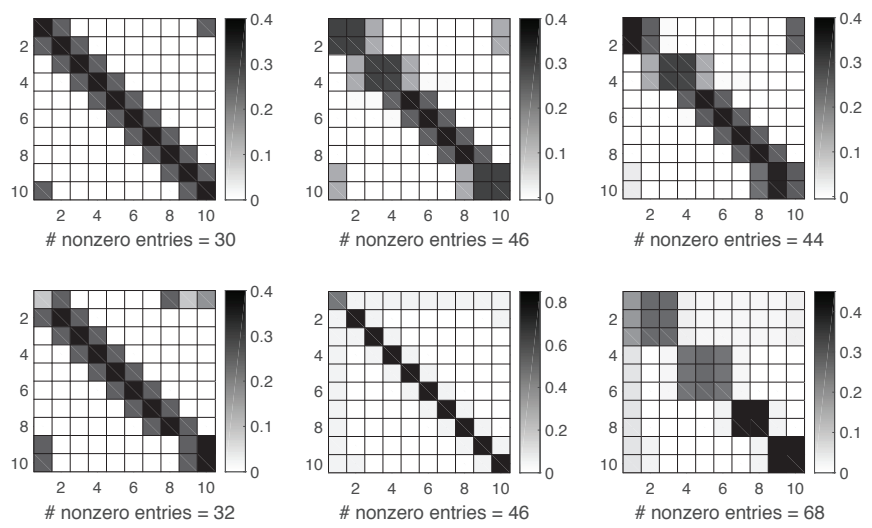

Fig. 4. (1st row) Sparsity pattern of matrices $\mathbf{P}, \boldsymbol{\Pi}_{\mathcal{M}} \mathbf{P}$ and $\boldsymbol{\Pi}_{\mathrm{ker}\left(\mathbf{M}_{1}\right)}^{\mathrm{ob}}$ considered in Figs. 2(a)-(c); (2nd row) Sparsity pattern of matrices $\mathbf{\Pi}_{\mathcal{M}, \mathcal{N}} \mathbf{P}, \mathbf{P}$ and $\mathbf{\Pi}_{\mathcal{M}} \mathbf{P}$ considered in Figs. 3(a)-(c).

for the constrained 2D rendezvous problem have validated the proposed theory.

In future works, we plan to extend our results to directed communication networks and characterize the convergence speed of our protocols We also aim at generalizing the proposed theory to time-varying and nonlinear constraints (e.g. by locally projecting the joint state onto the tangent space of the constraint manifold [20]). Lastly, for given a graph topology, we would like to check how restrictive is the rank condition in Theorem 7, for projection design. 


\section{REFERENCES}

[1] R. Olfati-Saber, J.A. Fax, and R.M. Murray. Consensus and Cooperation in Networked Multi-Agent Systems. Proc. of IEEE, 95(1):215233, 2007.

[2] M. Mesbahi and M. Egerstedt. Graph Theoretic Methods in Multiagent Networks. Princeton University Press, 2010.

[3] F. Bullo. Lectures on Network Systems. CreateSpace, 1st edition, 2018. With contributions by J. Cortes, F. Dörfler, and S. Martinez.

[4] D.V. Dimarogonas and K.J. Kyriakopoulos. On the Rendezvous Problem for Multiple Nonholonomic Agents. IEEE Trans. Automat. Contr., 52(5):916-922, 2007.

[5] B.A. Francis and M. Maggiore. Flocking and Rendezvous in Distributed Robotics. SpringerBriefs in Electrical and Computer Engineering. Springer, 2016.

[6] R. Olfati-Saber. Flocking for Multi-Agent Dynamic Systems: Algorithms and Theory. IEEE Trans. Automat. Contr., 51(3):401-420, 2006.

[7] N. Moshtagh and A. Jadbabaie. Distributed Geodesic Control Laws for Flocking of Nonholonomic Agents. IEEE Trans. Automat. Contr., 52(4):681-686, 2007.

[8] R. Sepulchre, D.A. Paley, and N.E. Leonard. Stabilization of Planar Collective Motion With Limited Communication. IEEE Trans. Automat. Contr., 53(3):706-719, 2008.

[9] K.-K. Oh, M.-C. Park, and H.-S. Ahn. A survey of multi-agent formation control. Automatica, 53:424-440, 2015.

[10] A. Franchi, P. Stegagno, and G. Oriolo. Decentralized multi-robot encirclement of a 3D target with guaranteed collision avoidance. Auton. Robot., 40(2):245-265, 2016.

[11] M. Schwager, D. Rus, and J.-J. Slotine. Unifying geometric, probabilistic, and potential field approaches to multi-robot deployment. Int. J. Robot. Res., 30(3):371-383, 2011.

[12] F. Morbidi, R.A. Freeman, and K.M. Lynch. Estimation and Control of UAV Swarms for Distributed Monitoring Tasks. In Proc. American Contr. Conf, pages 1069-1075, 2011.

[13] Y. Cao, W. Ren, and M. Egerstedt. Distributed containment control with multiple stationary or dynamic leaders in fixed and switching directed networks. Automatica, 48(8):1586-1597, 2012.

[14] M.M. Zavlanos, M. Egerstedt, and G.J. Pappas. Graph-Theoretic Connectivity Control of Mobile Robot Networks. Proc. IEEE, 99(9):15251540, 2011.

[15] K.M. Lynch, I.B. Schwartz, P. Yang, and R.A. Freeman. Decentralized Environmental Modeling by Mobile Sensor Networks. IEEE Trans. Robot., 24(3):710-724, 2008.

[16] S.S Kia, B. Van Scoy, J. Cortés, R.A. Freeman, K.M. Lynch, and S. Martínez. Tutorial on Dynamic Average Consensus: The Problem, its Applications, and the Algorithms. IEEE Contr. Syst. Mag., 39(3):40-72, 2019.

[17] A. Nedić, A. Ozdaglar, and P.A. Parrilo. Constrained Consensus and Optimization in Multi-Agent Networks. IEEE Trans. Automat. Contr., 55(4):922-938, 2010.

[18] U. Lee and M. Mesbahi. Constrained Consensus via Logarithmic Barrier Functions. In Proc. 50th IEEE Conf. Dec. Contr, pages 36083613, 2011.

[19] X. Wang and Z. Zhou. Projection-Based Consensus for ContinuousTime Multi-Agent Systems with State Constraints. In Proc. 54th IEEE Conf. Dec. Contr, pages 1060-1065, 2015.

[20] C. Lageman and Z. Sun. Consensus on spheres: convergence analysis and perturbation theory. In Proc. 55th IEEE Conf. Dec. Contr, pages 19-24, 2016.

[21] Z. Qiu, S. Liu, and L. Xie. Distributed constrained optimal consensus of multi-agent systems. Automatica, 68:209-215, 2016.

[22] A. Fontan, G. Shi, X. Hu, and C. Altafini. Interval Consensus for Multiagent Networks. IEEE Trans. Automat. Contr., 2019, in press.

[23] G. Shi, K.H. Johansson, and Y. Hong. Reaching an Optimal Consensus: Dynamical Systems That Compute Intersections of Convex Sets. IEEE Trans. Automat. Contr., 58(3):610-622, 2012.

[24] S. Jafarpour and F. Bullo. Synchronization of Kuramoto Oscillators via Cutset Projections. IEEE Trans. Automat. Contr., 64(7):2830-2844, 2019.

[25] L.E. Andersson, L. Imsland, E.F. Brekke, and F. Scibilia. On Kalman filtering with linear state equality constraints. Automatica, 101:467470, 2019

[26] D. Simon. Kalman filtering with state constraints: a survey of linear and nonlinear algorithms. IET Control Theory A., 4(8):1303-1318, 2010.

[27] A. Galántai. Projectors and Projection Methods, volume 6 of Advances in Mathematics. Springer, 2004.

[28] A. Ben-Israel and T.N.E. Greville. Generalized Inverses: Theory and Applications. Springer, 2nd edition, 2003.
[29] C. Godsil and G. Royle. Algebraic Graph Theory. Springer, 2001.

[30] D.S. Bernstein. Matrix Mathematics: Theory, Facts, and Formulas. Princeton University Press, 2nd edition, 2009.

[31] F. Blanchini and S. Miani. Set-Theoretic Methods in Control. Birkhäuser, 2nd edition, 2015.

[32] F. Morbidi. Subspace Projectors for State-Constrained Multi-Robot Consensus - Extended Version. Technical report, MIS Laboratory, Université de Picardie Jules Verne, Amiens, France, March 2020 [web]: mis.u-picardie.fr/ fabio/MorbidiTech-ICRA20.pdf.

[33] B. Siciliano, L. Sciavicco, L. Villani, and G. Oriolo. Robotics Modelling, Planning and Control. Advanced Textbooks in Control and Signal Processing. Springer, 2009 